\section{Current Issues}

Check for updates

\title{
Issues on Current HPV Vaccination in Korea and Proposal Statement
}

\author{
Kyoung Eun Lee
}

OPEN ACCESS

Received: Dec 7, 2019

Revised: Dec 11, 2019

Accepted: Dec 13, 2019

Corresponding author:

Kyoung Eun Lee

College of Nursing and Health Science, Texas A\&M University, 6300 Ocean Drive, Mail Unit

\#5805, Corpus Christi, TX 78414, USA.

Tel: +1-214-425-4414

Fax: +1-361-825-2484

E-mail: Kyoung.Lee@tamucc.edu

c) 2019 Korean Society of Women Health Nursing

This is an open access article distributed under the terms of the Creative Commons Attribution Non-Commercial License (https:// creativecommons.org/licenses/by-nc/4.0/) which permits unrestricted non-commercial use, distribution, and reproduction in any medium, provided the original work is properly cited.

ORCID iDs

Kyoung Eun Lee (D)

https://orcid.org/0000-0002-0881-1022

Conflict of Interest

The author declared no conflict of interest.
Assistant Professor, College of Nursing and Health Science, Texas A\&M University, Corpus Christi, TX, USA

Keywords: HPV vaccines; Cervical cancer

\section{ISSUES ON CURRENT HPV VACCINATION IN KOREA AND PROPOSAL STATEMENT}

Human papillomavirus (HPV) is the name of a group of viruses that cause skin infection. HPV is the major "sexually transmitted" virus that are responsible of $99 \%$ of cervical cancer [1]. In the U.S., most adult women go to see doctors for comprehensive annual health exam and the cervical cancer screening test (Papanicolaou [Pap] smear) is a must part of women's annual check-up. The Pap smear test is strongly recommended either to all women who started sexual intercourse regardless of age or any woman who is older than 21 regardless of previous sexual history. In the meantime, the test is not socially perceived nor recommended as a routine test for women in Korean until advanced age, causing high morbidity and mortality rate due to the failure of early detection and treatment for the cancer.

Cervical cancer can be prevented up to $90 \%$ by proper HPV vaccination. Since the HPV vaccine was developed and used worldwide, the cervical cancer rate has been consistently decreasing in the U.S. and many countries in Europe for last decade [1]. On the other hand, according to a recent report of Ministry of Health and Welfare [2], the incident of cervical cancer in Korea has been continuously increased for last 20 years. It was 44,000 in 1999 but became 103,000 in 2017, which is 2.5 times higher. Multiple factors contributed to the increase in combination including social/cultural stigma on preventive screenings, related public health policies, and health care delivery system in Korea. However, the largest problem is that the perceived significance of HPV vaccine among Korean is still very low compared to the U.S. and other developed European countries, resulting in low HPV vaccination rate and delayed its inclusion to the Korean National Immunization Program (NIP).

Most people in Korea have an idea that cervical cancer occurs in only for women of middle or old age, but another recent health statistics reported that the cervical cancer rate in 20 s and 30s in Korea has been consistently increased and it counts as more than $10 \%$ of total incidents as of 2016 [3]. Considering the current early age of women who start sexual activities compared to their parent's generation, there is an urgent need for health care providers and public health sectors in Korea to try to prevent HPV infection via proper HPV vaccination before women and men start any sexual relationship. In June 2016, Korea 
included free HVP vaccinations in its NIP. However, the HPV vaccine provided by NIP in Korea has not brought significant change as expected. There are several problematic issues to be discussed in timely manner. It is important to critically appraise those issues related to current NIP related to HPV vaccine and what we can do to make it better at this point.

The purpose of this issue paper is to thoroughly examine the critical issues in current HPV vaccination and its related NIP policies in Korea comparing to the U.S. and other developed countries and to explore the strategies and solution for increasing HPV vaccine rate and preventing the misuse of the vaccine.

\section{WHAT ARE THE CRITICAL ISSUES?}

\section{Lack of clear understanding of HPV, HPV infection and NIP covered HPV vaccines}

To better understand how the HPV vaccine works, you should first understand what the characteristics of HPV. HPV are very common virus and there are more than 100 different types. Not every HPV virus cause cancer and $80-90 \%$ of HPV infection is resolved by itself without any treatment within 1-2 years [4]. However, there are certain types of HPV, which are called as high-risk genetic group. Among those, type 16 and 18 are responsible for about $70 \%$ of cervical cancer, the rest (30\%) are caused by type $31,33,35,45,52,58$, and other rare types [1]. Among Korean women, it was reported that HPV type 52 and 58 are more prevalent than women in other ethnic groups [5]. In addition, the high-risk types of HPV can cause other cancers such as cancer in the oropharynx, anus, rectum, penis, vagina, and vulva [4]. The HPV virus is transmitted by sexual relationships. Therefore, the risk of HPV infection increases once people start sexual activities. This imposes the importance of getting HPV vaccine in early age, ideally 11 and 12 years old. In other words, getting HPV vaccine after marriage and childbirth in middle-aged or elderly years has minimal preventive effects against cervical cancer since they had already exposed to those various types of HPV. Nevertheless, many women in those age group in Korea are getting HPV vaccine by paying out of pocket money because their doctors strongly suggested its vaccination without providing properly information on the very limited scope of cancer prevention.

According to a recent report from World Health Organization [6], there are 67 countries who included HPV vaccine in their NIP. More than 2.7 million HPV vaccines were given in worldwide, supported by a safety review that the Global Advisory Committee on Vaccine Safety has provided [6]. Among those countries, 27 countries (e.g. the U.S. and Austria) are currently using only Gadasil 9 which has the highest cancer preventive rate (90\%) [7]. Korea has adopted the HPV vaccine to NIP in 2016, almost 10 year later than the U.S. and other developed counties. Despite its late start for the availability of free HPV vaccine, NIP in Korea has been providing free vaccine to extremely limited age group of only 12 -year-old girls (no one before or after 12 years old) and ignored the age group of 9 to 26 , which is the recommended range of HPV vaccination for proper preventive effects. Therefore, the NIP in Korea has minimal impacts on HPV vaccine rate among those age groups. Furthermore, Korean NIP offers Cervarix and Gadasil 4 vaccine, which have relatively low preventive rate $(70 \%$ and $80 \%)$ compared to Gadasil $9(90 \%)$.

It has been already 4 years since free HPV vaccination offered by NIP in Korea. However, there is serious lack of clear understanding of HPV, HPV infection, and NIP covered HPV vaccines among not only health care provider but the public in general. 


\section{Lack of clear understanding of the indications and differences among 3 HPV vaccine types: Cervarix, Gardasi ${ }^{\circledR}$ 4, and Gardasil $^{\circledR} 9$}

Many people, even health care providers, have limited understanding of the indications and differences among 3 available HPV vaccines. HPV vaccines are vaccines that prevent infection with certain types of causing cervical cancer. There are currently $3 \mathrm{HPV}$ vaccines available with different number of HPV coverage and prevention rate. Cervarix, the first available HPV vaccine, can prevent only 2 types of HPV (type 16 and 18) which is responsible of $70 \%$ of cervical cancer [8]. Later, Gadasil 4 became available and its cancer prevention rate increased up to $80 \%$ by covering 4 types in total (type 6,11,16, and 18). Gadasil 9, the most recent and effective yet expensive HPV vaccine, was approved by U.S. Food and Drug Administration in 2014. Since then, U.S. and many other European countries have been using only Gadasil 9 for HPV vaccination [7]. The Gadasil 9 , as implied by its name, covers 9 types of HPV (type 6, 11, 16, 18, 31, 33, 35, 52, and 58) and its cancer preventive rate is reported as higher than $90 \%$ [8]. Of course, health insurance in those countries completely cover the cost of Gadasil 9 for vaccinating "both man and women" in age of between 9 and 26. Unfortunately, this most effective vaccine was NOT included in Korean NIP and anyone who wants to get Gadasil 9 in Korea needs to pay $100 \%$ out of pocket money, ranged from 40,000 won to 90,000 won, for 3 doses. The high out-of-pocket cost of this vaccine has been identified major reason for many people in Korean not to consider getting HPV vaccination.

As previous mentioned, among Korean women, HPV type 52 and 58 are more prevalent than women in other ethnic groups [4] and Gadasil 9 is the only HPV vaccine that can prevent cervical cancer caused by those 2 types of HVP. Considering this clinical indication of proper HPV vaccination, it is a critical error for Korean NIP not to include Gadasil 9 as its free HPV vaccine in 2016.

\section{Lack of clear understanding of proper vaccination period and who needs HPV vaccine}

One of prevalent misconceptions about HPV vaccine among Korean is the importance of HPV vaccination among boys and men. You might think why boys and men need the HPV vaccine despite that they do not have cervix. HPV is a "sexually transmitted virus". Therefore, when you simply think about how the HPV is transmitted to women, it just makes perfect sense that boys and men need to prevent HPV infection by getting HPV vaccine before they start sexual activities. Besides, boys and men can also get health benefits from it by preventing HPV related anal and penile cancer as well as genital warts [9]. You need to acknowledge that vaccinating boys and men in recommended age range can significantly contribute to decrease the prevalence of HPV infection among girls and women.

According to the vaccine information statement issued by U.S. Center for Disease Control and Prevention (CDC) [10], HPV vaccine is recommended to "both men and women" in age of between 9 to 26. It also states that, although it may be given to up to 45 years old, but most people older than 26 years will not benefit from HPV vaccination [10]. The lack of clear understanding of this proper vaccination period among Korean mislead many Korean women in middle or older age group paid $100 \%$ out of pocket money to get the HPV vaccine simply because their doctors recommended it without proper information about the minimal/no benefits of HPV vaccination.

\section{WHAT WE NEED TO DO?}

Most of all, prompt change in health policies are required so that all 3 kinds of HPV vaccines (Cervarix, Gadasil 4, and Gadasil 9) can be covered by national health insurance in Korea for at 
least the HPV vaccine recommendation age group (age of 9 to 26). It is shameful that Korean NIP decided to provide free HPV vaccination almost 10 years later than the U.S. and other developed countries in Europe, but it is more shameful that Korean NIP takes an outdated action by offering only 2 HPV vaccines, which are NOT used any more in other developed countries, and to those girls who were born in only specific year (age 12). If she missed the HPV vaccine before December 31 of the offered year, she becomes not qualified for the free vaccine. This absurd NIP policy could sound so ridiculous for many others like me who have lived in different health care delivery system for several decades. Many might think, the decision to include HPV vaccine to the NIP looks like just another show-up business of Korean public health government sector, saying nothing more than "at least, we did something here". Doing something is NOT important when we are talking about public health people in the nation. "We do the things in right ways" should be more like it. To do it in right ways, Gadasil 9 which can prevent HPV types 52 and 58 (most prevalent cervical cancer-causing HPV type among Korean women) should be properly included in the NIP in Korea with extended covered range of age and should be available to the public at more affordable price.

And another significant problem regarding HPV vaccination in Korea is that, health care providers (e.g nurses and doctors) and health educators (e.g. public health nurse and school nurse) are minimally educated and have limited knowledge about HPV, HPV infection, and the effectiveness/clinical implication/safety of HPV vaccines. Maybe, the bigger problem is that there is serious lack of perception on how important it is to be professionally prepared to educate patients and public in general about the topics. As results, people, especially parents of teenagers, are not properly informed and have limited health resources to obtain appropriate knowledge about HPV risk and the various health benefits of getting HPV vaccine. Considering this, health researchers need to immediately investigate current knowledge level and lack of perceived importance of HPV vaccine among health care providers and educators in Korea as the first step. Then, systemic strategic plans aiming at increasing their knowledge and professional preparedness need to be developed in timely manners. Nurses are at the front line of public education about vaccination. It is also very important for professors of nursing program to integrate the knowledge about HPV and HPV vaccine into their course contents at early stage of program so that nursing students can properly educate patients, family, and community during their clinical practice as well as after graduation.

Health care providers and educators in clinical and academic settings need to take more active roles in educating young teenagers, their parents, and local public communities about the importance of getting HPV vaccine. Since HPV vaccines can be co-administered with the meningococcal conjugate vaccine or Tdap vaccine [6], nurses and doctors in primary care clinics can offer HPV vaccination when they visit the clinics for those other mandatory vaccines by emphasizing the importance of preventing HPV infection via getting vaccine early. To facilitate this, all of clinic providers and staffs should have proper training on how to successfully communicate with teenagers and their parents about HPV vaccination in more effective ways. In addition, starting with the front office, it is critical that everyone is on the same page when it comes to proper vaccination practices, recommendations, and how to answer parents' questions about HPV vaccine.

Korean health government sectors like Korean CDC should be able to provide health care providers and educators with most reliable evidence-based practice guidelines and to update it on regular basis. And differentiated HPV vaccine statements using age-appropriate languages need to be developed for more effective educational impact on different age group 
of people. In addition, broad spectrum of educational opportunities should be given to teachers and school nurses of all elementary, middle, and high schools, especially in rural areas, so that they can have clear understanding of how to prevent HPV infection and why getting vaccination is so critical for students.

Teenagers and their parents themselves should be properly motivated to learn about HPV and the vaccine. We need to utilize different formats of mass media as educational tools to deliver knowledge about HPV and HPV vaccine more effectively to teenager groups. For example, the education can be delivered via popular web-toons, music video filmed by K-POP idol groups, and public speech by Korean celebrities rather than by politicians or public health educators with no sense of humor. In addition, more aggressive strategic plans should be discussed to deliver the importance of HPV vaccination to the public in general using creative ways for actual impacts on their health seeking behaviors.

Lastly, the misuse/abuse of HPV vaccine as a way of making money among health care providers should be deterred by assuring (legal) enactment. Offering HPV vaccine to the women older than 45, who do not have any benefits from getting HPV, is wrongful deception, intended to have financial or personal gain. It is "fraudulent behavior" by its definition. The wrongful offer of HPV vaccine to those age group should not be considered as just unethical behaviors but be considered as serious fraudulent misconduct requiring corresponding penalty imposed by government sector. I have heard from many relatives and friends in late 40 s that they got 3 doses of 'a kind of HPV vaccine' recommended by their doctor, ended up with paying at least 400,000-900,000 won because it has never been covered by Korean national insurance. None of those was properly informed about the details about HPV vaccine and its clinical implications. They said, they got the HPV vaccine because the doctors told them to get it to prevent the cervical cancer. As result, those women have the misleading idea that they would not have a cervical cancer in their later life and do not need to do any pap smear test. This can bring out serious long-term impacts on decreasing screening rate for cervical cancer. This issue should be brought to immediate attention of the public and government sectors.

This issue paper was aiming at examining several critical issues in current available HPV vaccination in Korea and its related NIP policies comparing to the U.S. and other developed countries. In addition, the author tried to provide an insight for developing proper strategies and solutions for the future to make health care providers and educator professionally prepared and to prevent the misuse of the vaccine.

\section{REFERENCES}

1. Strategic Advisory Group of Experts (SAGE). Working group on potential contribution of HPV vaccines and immunization towards cervical cancer elimination [Internet]. Geneva: World Health Organization; 2018 [cited 2019 Nov 20]. Available from: https://www.who.int/immunization/policy/sage/sage_wg_ hpv_2018/en/.

2. Ministry of Health and Welfare. Statistics of cancer morbidity and mortality in Korea [Internet]. Seoul: Ministry of Health and Welfare; 2016 [cited 2019 Nov 20]. Available from: http://www.index.go.kr/potal/ main/EachDtlPageDetail.do?idx_cd=2770.

3. Health Insurance Review \& Assessment Service. Cervical cancer in 20s and 30s [Internet]. Seoul: Health Insurance Review \& Assessment Service; 2016 [cited 2019 Nov 19]. Available from: http://www.hira.or.kr/ bbsDummy.do?pgmid=HIRAA020041000100\&brdScnBltNo=4\&brdBltNo=9153\&pageIndex=1 . 
4. National Cancer Institute. HPV and cancer [Internet]. Bethesda, MD: National Cancer Institute; 2019 [cited 2019 Nov 20]. Available from: https://www.cancer.gov/about-cancer/causes-prevention/risk/ infectious-agents/hpv-and-cancer.

5. Lee BR. Human papilloma virus type 52 \& 58: causing higher rate of cervical cancer in Korea [Internet]. Seoul: ChosunMedia; 2019 [cited 2019 Nov 20]. Available from: http://health.chosun.com/site/data/ html_dir/2016/09/20/2016092001976.html.

6. World Health Organization. Safety update of HPV vaccines [Internet]. Geneva: World Health Organization; 2017 [cited 2019 Nov 21]. Available from: https://www.who.int/vaccine_safety/committee/ topics/hpv/June_2017/en/.

7. Lee HG. $90 \%$ of HPV prevention rate of Gadasil 9, selected by world as national immunization program [Internet]. Seoul: PhamNews; 2019 [cited 2019 Dec 1]. Available from: http://www.pharmnews.com/ news/articleView.html?idxno=97508.

8. National Cancer Institute. Human papillomavirus (HPV) vaccines [Internet]. Bethesda, MD: National Cancer Institute; 2019 [cited 2019 Nov 20]. Available from: https://www.cancer.gov/about-cancer/causesprevention/risk/infectious-agents/hpv-vaccine-fact-sheet.

9. Center for Disease Control and Prevention. HPV (human papillomavirus) vaccine: what you need to know [Internet]. Atlanta, GA: Center for Disease Control and Prevention; 2019 [cited 2019 Nov 19]. Available from: https://www.cdc.gov/vaccines/hcp/vis/vis-statements/hpv.html.

10. Center for Disease Control and Prevention. HPV vaccine recommendations [Internet]. Atlanta, GA: Center for Disease Control and Prevention; 2016 [cited 2019 Nov 19]. Available from: https://www.cdc. gov/vaccines/vpd/hpv/hcp/recommendations.html. 